\title{
BIOINFORMATICS 2014
}

$5^{\text {TH }}$ INTERNATIONAL CONFERENCE ON

BIOINFORMATICS MODELS, METHODS AND ALGORITHMS

\section{PROCEEDINGS}

ESEO, ANGERS, LOIRE VALLEY, FRANCE

3 - $6 \mathrm{MARCH}, 2014$

THIS CONFERENCE IS PART OF

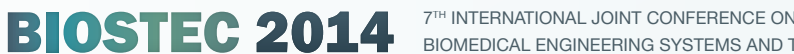

SPONSORED BY: $\quad$ CO-ORGANIZED BY:

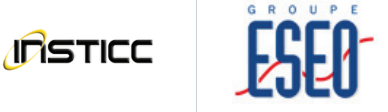

TECHNICAL CO-SPONSORSHIP BY:

- 


\title{
BIOINFORMATICS 2014
}

\author{
Proceedings of the \\ International Conference on \\ Bioinformatics Models, Methods and Algorithms
}

\author{
ESEO, Angers, Loire Valley, France
}

$$
3 \text { - } 6 \text { March, } 2014
$$

Sponsored by

INSTICC - Institute for Systems and Technologies of Information, Control and Communication

$$
\begin{gathered}
\text { Co-organized by } \\
\text { ESEO }
\end{gathered}
$$

Technical Co-sponsorship by

ESEM - European Society for Engineering and Medicine

BMES - Biomedical Engineering Society

IEEE EMBS - IEEE Engineering in Medicine and Biology Society

In Cooperation with

ACM SIGART - ACM Special Interest Group on Artificial Intelligence

ACM SIGBio - ACM Special Interest Group on Bioinformatics, Computational Biology, and Biomedical Informatics

AAAI - Association for the Advancement of Artificial Intelligence

EUROMICRO

ISfTeH - International Society for Telemedicine \& eHealth

Supported by

Région Pays de la Loire

Conseil Général de Maine et Loire

Ville d'Angers

Angers Loire Métropole

Angers Loire Tourisme 
Copyright @ 2014 SCITEPRESS - Science and Technology Publications All rights reserved

Edited by Oscar Pastor, Christine Sinoquet, Guy Plantier, Tanja Schultz, Ana Fred and Hugo Gamboa

\section{Printed in Portugal}

ISBN: 978-989-758-012-3

Depósito Legal: 370690/14

http://www.bioinformatics.biostec.org

bioinformatics.secretariat@insticc.org 


\title{
Studies of Mutation Accumulation in Three Codon Positions using Monte Carlo Simulations and Metropolis-Hastings Algorithm
}

\author{
Małgorzata Grabińska, Paweł Błażej and Paweł Mackiewicz \\ Department of Genomics, Faculty of Biotechnology, University of Wroctaw, Wroctaw, Poland \\ mwcleo@gmail.com,blazej@smorfland.uni.wroc.pl,pamac@smorfland.uni.wroc.pl
}

Keywords: Codon, Evolution, Metropolis-Hastings Algorithm, Monte Carlo Simulations, Mutation, Nucleotide Composition, Protein Coding Sequence, Rate Matrix, Selection, Substitution, Transition, Transversion.

\begin{abstract}
Protein coding sequences are characterized by specific nucleotide composition in three codon positions as a result of mutational and selection pressures. To analyse the impact of mutations and different transition/transversion ratio on three codon position in protein coding sequences, we elaborated a model of genome evolution based Monte Carlo simulation. Selection was applied against stop translation codons and modified Metropolis-Hastings algorithm to maintain typical nucleotide composition of particular codon positions. The simulations were performed on genomes consisting of bacterial gene sequences. We used a series of nucleotide substitution matrices assuming different transition/transversion ratio and nucleotide stationary distribution characteristic of the real mutational pressure. The simulations showed exponential decrease in the number of eliminated genomes with the growth of the transition/transversion ratio. The same trend was also observed both for accepted and to lesser extent for rejected mutations. The third codon positions much more mutations accepted than rejected because of very similar composition to the mutational stationary distribution, whereas the first positions accumulated the smallest number of mutations and rejected the most as a result of strong selection on its nucleotide composition. The obtained results showed different response of three codon positions on mutational pressure related with their characteristic nucleotide composition.
\end{abstract}

\section{INTRODUCTION}

One of characteristic features of protein coding sequences resulting from their coding and functional requirements is their triplet (codon) structure, which is related to a specific nucleotide composition of three codon positions (Wong and Cedergren, 1986; Anderson and Kurland, 1990; Zhang and Zhang, 1991; Gutierrez et al., 1996; Cebrat et al., 1997a; Cebrat et al., 1998; Wang, 1998). There are two forces, mutation pressure and selection constraints, which can change or maintain this composition (Frank and Lobry, 1999).

The first two codon positions are usually subjected to strong selective constraints although some influence of replication-associated mutational pressure was also observed (McLean et al., 1998; Cebrat et al., 1999; Mackiewicz et al., 1999a; Tillier and Collins, 2000; Kowalczuk et al., 2001b). Generally, the first codon positions of protein coding sequences are rich in purines, guanine $(\mathrm{G})$ and adenine (A), whereas the second positions are poor in guanine and contain more cytosine (C) and adenine. The dom- inance of purines, and particularly guanine in the first codon position and their deficiency in the second position can ensure the correct reading frame of transcripts during translation by interaction of nucleotides in the first codon positions of mRNA with also periodically distributed cytosines in rRNA (Trifonov, 1987; Lagunez-Otero and Trifonov, 1992; Trifonov, 1992). In support of this, highly expressed genes are characterized by increased usage of codons starting from guanine and to lesser extent from adenine, which does not depend on the overall $\mathrm{G}+\mathrm{C}$ content in the genome (Gutierrez et al., 1996; Pan et al., 1998; Akashi, 2003; Das et al., 2005). This composition reflects also the frequent usage of acidic amino acids coded by GAN codons (Karlin and Mrazek, 1996) as well as glycine, alanine and valine in coded proteins (Karlin et al., 1992). It was found that these amino acids are very common in products of highly transcribed genes (Jansen and Gerstein 2000, Akashi 2003, Marin et al. 2003). The excess of purines in the coding sequences (Shepherd, 1981; Smithies et al., 1981; Karlin and Burge, 1995; Cebrat et al., 1997b; Freeman et al., 1998) was also explained by their less susceptibility to 
mutations than pyrimidines (Hutchinson, 1996). During transcription process, the sense strand of genes stays longer in the single-stranded state. Therefore, it is more exposed than the antisense strand, which is preferably repaired and protected by proteins (Mellon and Hanawalt, 1989; Hanawalt, 1991). Changes in the second position in codons are generally more conserved than in the first one because mutations in the former more often lead to changes in hydrophobicity and polarity of coded amino acid residues.

On the other hand, the third codon positions are most of all subjected to accumulations of mutations because most nucleotide substitutions in these sites usually do not change coded amino acid residues or their properties. However, not all substitutions are necessarily neutral. Some preferences in usage of synonymous codons (i.e. coded the same amino acid) were observed in highly expressed genes, which is positively correlated with tRNA content in cells and the rate of translation (Ikemura, 1981; Ikemura, 1985; Bennetzen and Hall, 1982; Sharp and Cowe, 1991; Kanaya et al., 1999). The third codon position are usually rich in pyrimidines, particulary in thymine (T), probably as a result of the most frequent point mutation, deamination of cytosine and its homologue 5-methylcytosine to uracile, which finally leads to substitution C to T (Echols and Goodman, 1991; Lindahl, 1993; Kreutzer and Essigmann, 1998).

There are two types of point mutations happening in protein coding sequences: transitions (substitution between the same chemical types of nucleotides, between purines and between pyrimidines) and transversions (substitution between the different types of nucleotides, between purines and pyrimidines). Transitions are usually several times more often observed in real sequences than transversions although the expected ratio is 1:2 if all substitutions are equally likely (Wakeley, 1996). This bias results from higher rate of chemical changes between nucleotides with the similar structure and more common transition substitutions introduced during replication of genetic material. Moreover, transitions more rarely cause changes in coded amino acids or their properties, therefore are more often accepted than transversions.

To study the influence of mutations and different transition/transversion rate on accumulation of substitutions in three codon position of protein coding sequences, we elaborated Monte Carlo simulation model of genome evolution. As a selection module, we applied selection against occurrence of stop translation codons and a modified Metropolis-Hastings algorithm to keep nucleotide composition characteristic of a given codon position by acceptance or rejection of introduced mutations.

\section{MATERIALS AND METHODS}

The simulations were carried out for two million steps on the population of 72 individuals that represented protein coding sequences from bacterial genome of Borrelia burgdorferi. This genome is very suitable for mutation simulation studies (Kowalczuk et al., 1999; Błażej et al., 2012) because shows very strong compositional bias related to differently replicated leading/lagging DNA strands (McInerney, 1998; Mackiewicz et al., 1999b). Moreover, it has the determined mutational pressure associated with DNA replication (Kowalczuk et al., 2001a). In our simulation, each individual consisted of 333 gene sequences, with the total length of $353,035 \mathrm{bp}$, lying on the leading strand. The sequences and their annotations were downloaded from NCBI database (http://www.ncbi.nlm.nih.gov).

Table 1: The substitution rate matrix $P$ corresponding to HKY85 model, used in simulations. A nucleotide in the column is substituted by a nucleotide in the row. $\pi_{x}$ is the stationary frequency of a given nucleotide, whereas $\alpha$ corresponds to the transition rate.

\begin{tabular}{|c|c|c|c|c|}
\hline & $\mathbf{A}$ & $\mathbf{C}$ & $\mathbf{G}$ & $\mathbf{T}$ \\
\hline $\mathbf{A}$ & - & $\pi_{C}$ & $\alpha \pi_{G}$ & $\pi_{T}$ \\
\hline $\mathbf{C}$ & $\pi_{A}$ & - & $\pi_{G}$ & $\alpha \pi_{T}$ \\
\hline $\mathbf{G}$ & $\alpha \pi_{A}$ & $\pi_{C}$ & - & $\pi_{T}$ \\
\hline $\mathbf{T}$ & $\pi_{A}$ & $\alpha \pi_{C}$ & $\pi_{G}$ & - \\
\hline
\end{tabular}

The applied Monte Carlo simulations consisted of two stages: mutation of gene sequences and selection of individuals in population. The mutations were introduced into the sequences according to the Poisson process with average equal to one mutation per genome. Nucleotide substitutions (mutations) were generated by a probability matrix $P$ (Table 1 ) described by the HKY85 model (Hasegawa et al., 1985). This model distinguished transversion and transition rates as well as assumed that a given substitution was proportional to the stationary frequency of nucleotide $\pi_{x}$ that was created by this substitution. The stationary distribution of nucleotides was the same as for the empirical matrix describing mutational pressure for the leading strand in B. burgdoferi genome (Table 2), which was also used in these simulation for comparison.

We decided to use the modified model of nucleotide substitution because it enabled easy implementation of various transition rates $\alpha$ and, simultaneously, inclusion of the assumed stationary distribution. We tested different values of $\alpha$ from 0.1 to 10 with the step of 0.1 . For all cases, transversion rate was fixed to 1 and defined only from frequencies of nucleotides under the stationary distribution $\pi$. 
Table 2: The uniformized substitution matrix describing real mutational pressure for the leading DNA strand in the B. burgdorferi genome (Kowalczuk et al., 2001a). A nucleotide in the column changes to a nucleotide in the row with the given probability.

\begin{tabular}{|c|c|c|c|c|}
\hline & $\mathbf{A}$ & $\mathbf{C}$ & $\mathbf{G}$ & $\mathbf{T}$ \\
\hline $\mathbf{A}$ & 0.81 & 0.02 & 0.07 & 0.10 \\
\hline $\mathbf{C}$ & 0.07 & 0.62 & 0.05 & 0.26 \\
\hline $\mathbf{G}$ & 0.16 & 0.01 & 0.71 & 0.12 \\
\hline $\mathbf{T}$ & 0.07 & 0.03 & 0.03 & 0.87 \\
\hline
\end{tabular}

Every substitution rate matrix was transformed to jump probability matrix using uniformization method (Tijms, 2003), see Table 3 as an example. This approach is generally used to change the original continuous in time Markov process with non-identical leaving rates into an equivalent of stochastic process where transition between each states are generated by Poisson process with the same fixed rate. This method is very useful in the simulation of multidimensional Markov processes.

Table 3: The uniformized substitution matrix for the HKY85 model assuming the transition/transversion ratio $\alpha=1.1$ as for the real mutational matrix. A nucleotide in the column changes to a nucleotide in the row with the given probability.

\begin{tabular}{|c|c|c|c|c|}
\hline & $\mathbf{A}$ & $\mathbf{C}$ & $\mathbf{G}$ & $\mathbf{T}$ \\
\hline $\mathbf{A}$ & 0.79 & 0.02 & 0.07 & 0.12 \\
\hline $\mathbf{C}$ & 0.07 & 0.64 & 0.03 & 0.26 \\
\hline $\mathbf{G}$ & 0.17 & 0.01 & 0.70 & 0.12 \\
\hline $\mathbf{T}$ & 0.08 & 0.03 & 0.03 & 0.86 \\
\hline
\end{tabular}

Two types of selection were applied. One was against occurrence of termination translation codons. If one of three possible stop codons occurred inside a given protein coding sequence then the individual was removed from the population and replaced by another. The second type of selection was for maintenance of characteristic nucleotide composition in each of three positions in codon. To do so, we applied for every codon position a modified acceptancerejection method based on Metropolis-Hastings (MH) algorithm (Chib and Greenberg, 1995). In contrast to the original $\mathrm{MH}$ algorithm that generates a sequence of random samples from a stationary distribution $\pi$, we computed acceptance probability for each nucleotide substitution $a_{x y}$ using proposal transition probabilities and the assumed stationary distribution of nucleotides in three codon positions, separately:

$$
a_{x y}=\min \left(\frac{\pi_{y} q_{y x}}{\pi_{x} q_{x y}}, 1\right), \pi_{x} q_{x y}>0,
$$

where $\pi_{x}, x \in\{A, C, T, G\}$ is frequency of nucleotides in particular codon positions of protein coding sequences, whereas $q_{x y}$ are transition probabilities from matrix $P$ which generates mutation process (see Table 4 as an example).

Table 4: The acceptance-rejection matrices for three codon positions based on the HKY85 matrix assuming the transition/transversion ratio $\alpha=1.1$.

the first codon position
\begin{tabular}{|c|c|c|c|c|}
\hline & $\mathbf{A}$ & $\mathbf{C}$ & $\mathbf{G}$ & $\mathbf{T}$ \\
\hline $\mathbf{A}$ & 1 & 1 & 1 & 0.39 \\
\hline $\mathbf{C}$ & 0.62 & 1 & 1 & 0.25 \\
\hline $\mathbf{G}$ & 0.52 & 0.84 & 1 & 0.21 \\
\hline $\mathbf{T}$ & 1 & 1 & 1 & 1 \\
\hline
\end{tabular}

the second codon position

\begin{tabular}{|c|c|c|c|c|}
\hline & $\mathbf{A}$ & $\mathbf{C}$ & $\mathbf{G}$ & $\mathbf{T}$ \\
\hline $\mathbf{A}$ & 1 & 1 & 0.91 & 0.64 \\
\hline $\mathbf{C}$ & 0.39 & 1 & 0.36 & 0.25 \\
\hline $\mathbf{G}$ & 1 & 1 & 1 & 0.70 \\
\hline $\mathbf{T}$ & 1 & 1 & 1 & 1 \\
\hline
\end{tabular}

the third codon position

\begin{tabular}{|c|c|c|c|c|}
\hline & $\mathbf{A}$ & $\mathbf{C}$ & $\mathbf{G}$ & $\mathbf{T}$ \\
\hline $\mathbf{A}$ & 1 & 1 & 1 & 1 \\
\hline $\mathbf{C}$ & 0.84 & 1 & 0.88 & 0.85 \\
\hline $\mathbf{G}$ & 0.96 & 1 & 1 & 0.96 \\
\hline $\mathbf{T}$ & 0.99 & 1 & 1 & 1 \\
\hline
\end{tabular}

Additionally, the acceptance of substitution was determined by a random variable $U$ with uniform distribution with the range $[0,1]$. If $U>a_{x y}$, the substitution of $x$ by $y$ was rejected, otherwise it was accepted. It allowed to keep characteristic nucleotide composition in three codon position during simulations. For instance, if $\pi_{y} q_{y x}>\pi_{x} q_{x y}$, it indicated that changes from nucleotide $y$ to $x$ were too often than from nucleotide $x$ to $y$, then the move from nucleotide $x$ to new state $y$ was accepted. An individual in which the substitution was rejected, was 'killed' and replaced by another from the population.

\section{RESULTS AND DISCUSSION}

\subsection{Nucleotide Composition in Three Codon Positions}

Analysed protein coding sequences show characteristic nucleotide composition in three codon positions (Table 5). The first position is significantly rich in purines, adenine and guanine. However, it should be noticed that guanine is two times more frequent in this position than in others. The second position has generally more adenine and thymine with comparable frequencies although cytosine reaches the highest 
usage just in this position. The third position is also AT-rich but thymine significantly dominates. Interestingly, the composition of the third position is strikingly similar to the stationary distribution of empirical mutational matrix, whereas the composition of the first position significantly differs. It strongly prefers purines. It indicates that the third codon position is subjected to the weakest selection pressure then freely accumulates nucleotide substitutions resulting from mutations. On the other hand, the global composition of the first position is the least susceptible to the mutational pressure and is under the strongest selection.

Table 5: Nucleotide frequency for three positions in codon and stationary distribution for empirical mutational matrix.

\begin{tabular}{|c|c|c|c|c|}
\hline & A & C & G & T \\
\hline stationary & 0.32 & 0.06 & 0.14 & 0.48 \\
\hline 1st position & 0.37 & 0.11 & 0.30 & 0.22 \\
\hline 2nd position & 0.35 & 0.17 & 0.14 & 0.34 \\
\hline 3rd position & 0.31 & 0.07 & 0.14 & 0.48 \\
\hline
\end{tabular}

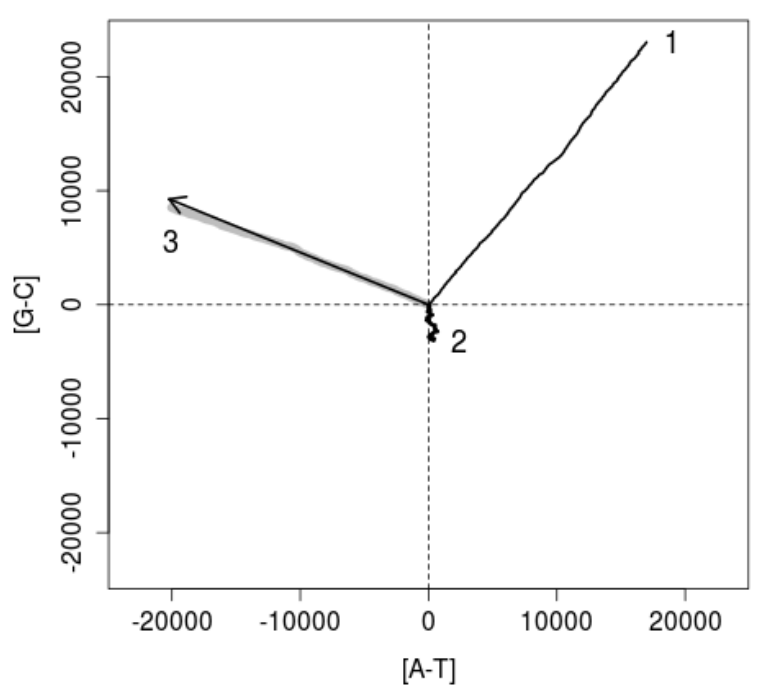

Figure 1: DNA walks, a graphical representation of nucleotide composition in three codon position of protein coding sequence from $B$. burgdorferi. The walk starts at the first nucleotide in the fixed codon position and jumps every third nucleotide to the last one. Every jump begins at the origin of a Cartesian plane and is associated with a unit shift, which depends on the nucleotide visited during the walk. The shift is $(0 ; 1)$ for guanine, $(1 ; 0)$ for adenine, $(0 ;-1)$ for cytosine, and $(-1 ; 0)$ for thymine. The vector indicates the stationary distribution of empirical mutational matrix.

The compositional trends are very well visualised by DNA walks (Figure 1), which are graphical representation of nucleotide composition in an analysed sequence (Cebrat and Dudek, 1998; Cebrat et al., 1998). The longest walk is clearly visible in the first codon position, which indicates the strongest compositional trend, i.e. strong preference of some nucleotides (here purines) than other. The clear trend is also in the third position, which very well matches the stationary composition generated by the empirical mutational matrix and shows excess of guanine over cytosine and thymine over adenine. On the other hand, the weakest trend is in the second position, which indicates that there are no special preferences in nucleotide occurrence in this position. It means that this position has more balanced frequency of complementary nucleotides, adenine vs. thymine and guanine vs. cytosine.

\subsection{Simulations of Mutation and Selection Processes}

Simulations for different transition/transversion ratios (calculated from elements of uniformised probability matrices) showed that the mean number of individuals eliminated from populations decreased in exponential manner with the increase of the ratio (Figure 2). It indicates a positive effect of the excess of transitions over transversions on genome survival.

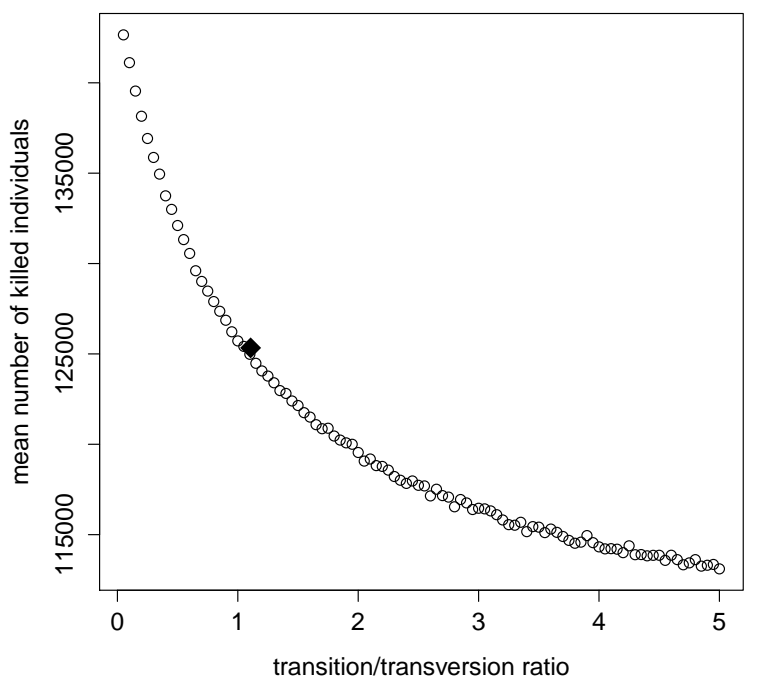

Figure 2: Mean number of eliminated individuals from population in the relationship to transition/transversion ratio. The black diamond indicates the empirical mutational matrix.

It is in agreement with the fact that transversions are more harmful by changing of coded amino acid than transition in protein coding sequences. The empirical matrix appeared very similar in the number of eliminated genomes to the HKY85 matrix assuming the same transition/transversion ratio 1.1. It seems that the applied HKY85 model is very good approximation of the real mutational matrix (please compare 
Table 3 and Table 2).

The mean number of accepted mutations in all codon positions was more than two times higher than the rejected ones (Figure 3). The number of both mutation types decreased with growth of transition/transversion ratio although the fall was larger for the accepted mutations. However, in both cases, the decrease became weaker and finally stabilized for higher transition/transversion ratios. It indicates that the increase in the ratio is not necessary to significantly diminish the rejected mutations. Interestingly, the values obtained for empirical matrix were very similar to the HKY85 model with the similar transition/transversion ratio. Genomes in the simulations with the real matrix accepted only slightly less mutations than in the case of the HKY85 model.

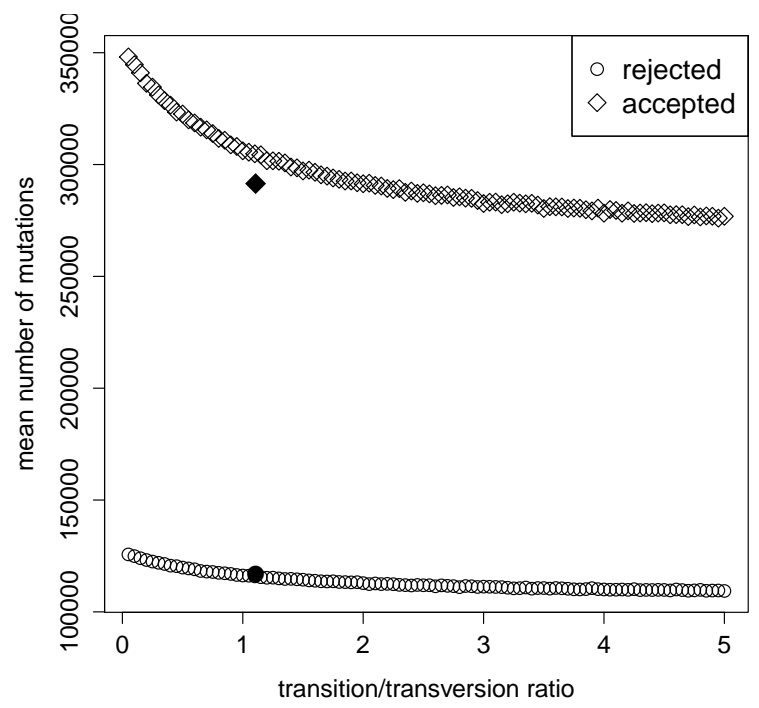

Figure 3: Mean number of rejected and accepted mutations in the relationship to transition/transversion ratio. The black circle and diamond indicate the empirical mutational matrix.

The mean of accepted mutations exceeded the number of rejected ones in three codon positions for tested values of transition/transversion ratio (Figure 4). The greatest difference between these numbers was for the third codon positions. The mutations were most frequently accepted and most rarely rejected in these positions. It results from very high similarity between nucleotide composition of these positions with the stationary distribution of the applied mutational matrix (Table 5, Figure 1). It indicates that the third codon positions are subjected to the weakest selection for the nucleotide composition and can quite freely accumulated mutations. Actually, they very well reflect mutational pressure associated with replication (McLean et al., 1998; Cebrat et al., 1999). On the other hand, the first codon positions accumulated the smallest number of mutations and rejected the most in comparison to other positions. Interestingly, the number of accepted and rejected mutations in the first codon positions became very similar when transition/transversion ratio declined. These strong restrictions on mutation accumulation in the first positions in our simulations result from the substantial compositional trend in these positions (Figure 1), which significantly deviates from the composition generated by the applied mutational pressure.

As it was reviewed in the Introduction, this specific composition is strongly related with various selection constraints on coding function of protein gene sequences. The number of mutations for the second positions had intermediate values between the first and third positions. In real sequences the second position usually is more conserved than the first one because substitutions in it always change coded amino acid and very often its physicochemical properties. However, our simulation considered only the effect of selection on nucleotide compositions but not restrictions on amino acid substitution. Thus our results suggest that the selection on nucleotide composition is weaker in the second codon position than the first one.

The relationship between the mean number of accepted or rejected mutations and transition/transversion ratio appeared different for three codon positions (Figure 4). Similarly to the case of mutations calculated for all positions (Figure 3), the number of accepted mutations for third and second codon positions declined rapidly with transition/transversion ratio and then begun stabilised for large values of ratio. However, the number of accepted mutations for first positions was stable and did not depend on the ratio. On the other hand, the exponential decrease was observed for the number of rejected mutations in these positions. In the remaining cases, the number of rejected mutations did not seem to depend from the transition/transversion ratio. Only a small increase in the number of rejected mutations was observed for the second and third codon positions. All the results about the number of accepted and rejected mutations suggest that excess of transitions over transversions has positive effect on maintenance of nucleotide composition characteristic of the first codon position, whereas negative in the case of other codon positions.

The differences between numbers of accepted and rejected mutations for the empirical matrix and the corresponding HKY85 model were generally very small (Table 6). The largest deviations were observed for the third codon positions especially for the number of rejected mutations. The empirical matrix rejected 


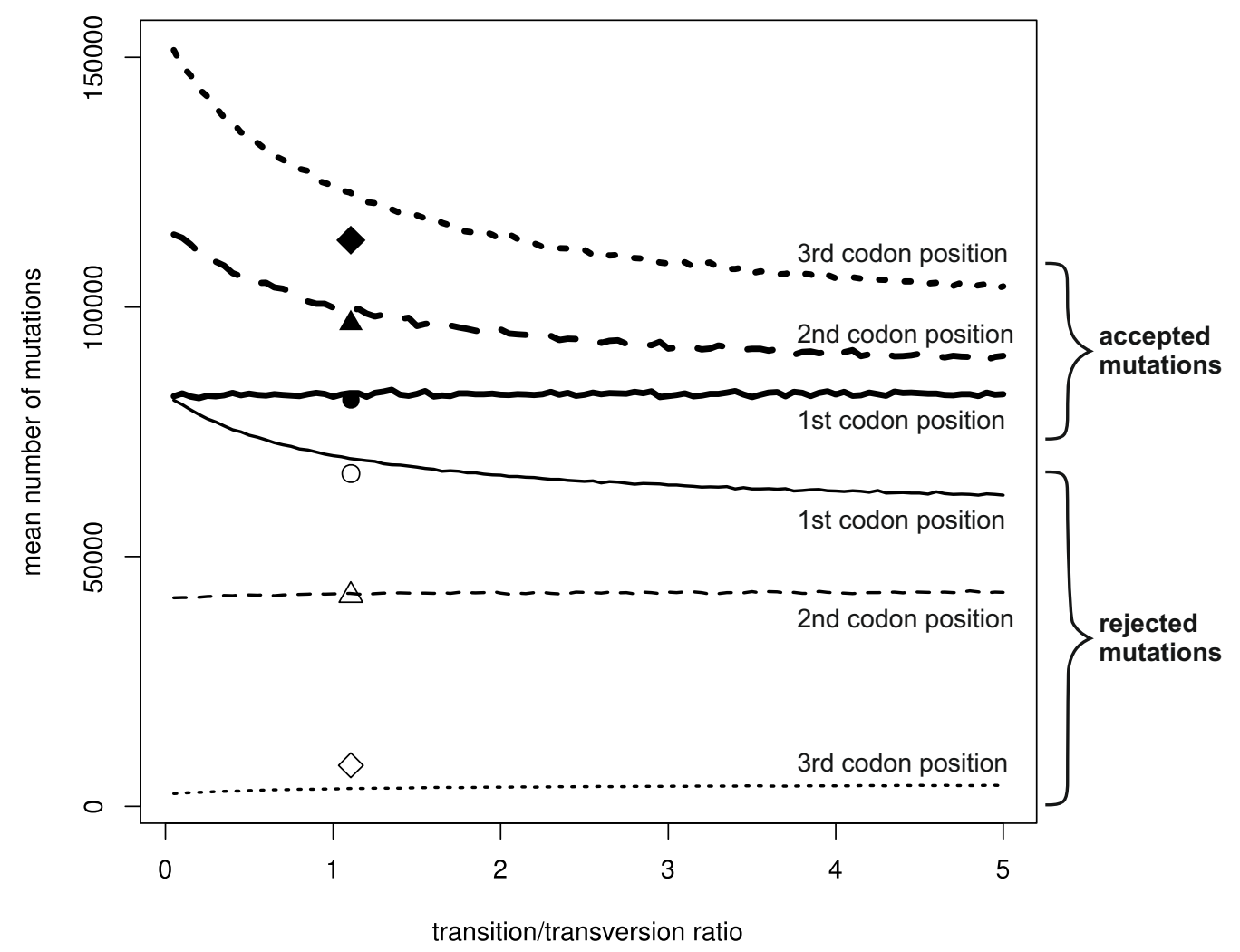

Figure 4: Mean number of rejected and accepted mutations in the relationship to transition/transversion ratio for three codon positions. The diamond, triangle and circle symbols indicate the empirical mutational matrix.

two times more mutations than the HKY85. Nevertheless, the number of these mutations for the third codon positions were much smaller (five to almost twenty times) in comparison to other positions.

Table 6: The number of accepted and rejected mutations in three codon positions for the empirical matrix and the HKY85 matrix assuming the transition/transversion ratio $\alpha=1.1$.

\begin{tabular}{|c|c|c|c|c|}
\hline & \multicolumn{2}{|c|}{ accepted } & \multicolumn{2}{c|}{ rejected } \\
\hline & empirical & HKY85 & empirical & HKY85 \\
\hline 1st & 81398 & 82757 & 66631 & 69663 \\
\hline 2nd & 96715 & 99078 & 42260 & 42627 \\
\hline 3rd & 113363 & 122957 & 8238 & 3580 \\
\hline
\end{tabular}

\subsection{CONCLUSIONS}

The obtained results showed that excess of transition over transversion in mutational pressure is generally profitable for the studied genome because mean number of eliminated individuals decreased exponentially with the growth of transition/transversion ratio (Figure 2). It is well-known that transversions are more harmful than transitions because they more frequently change coded amino acid. However, the presented results are not trivial because the simulations did not consider any selection on coded amino acids. Instead of that, we applied independent selection on nucleotide composition in three codon positions. The results indicate that not only genetic code and amino acid composition but also nucleotide composition typical of the first codon positions are optimized for high transition/transversion ratio. Moreover, these codon positions appeared most conserved because accepted the least and rejected the largest number of mutations. Interestingly, the second codon positions usually considered conserved according to the effect on amino acid substitution were more tolerant on mutation accumulation in our simulations. Because the applied model considered selection on nucleotide composition, it seems that maintenance of this composition by selection is more important for the first codon positions than for the second ones. It would be interesting to check if these conclusions are universal and valid for other genomes. 


\section{REFERENCES}

Akashi, H. (2003). Translational selection and yeast proteome evolution. Genetics, 164:1291-1303.

Anderson, S. G. E. and Kurland, C. G. (1990). Codon preferences in free-living microorganisms. Microbiol. Rev., 54:198-210.

Bennetzen, J. L. and Hall, B. D. (1982). Codon selection in yeast. J Biol Chem, 257(6):3026-3031.

Błażej, P., Mackiewicz, P., and Cebrat, S. (2012). Simulation of bacterial genome evolution under replicational mutational pressures. In Proceedings of the BIOSTEC 2012, 5th International Joint Conference on Biomedical Engineering Systems and Technologies Bioinformatics 2012, International Conference on Bioinformatics Models, Methods and Algorithms, Vilamoura, Algarve, Portugal, 1-4 February, pages 51-57.

Cebrat, S. and Dudek, M. (1998). The effect of DNA phase structure on DNA walks. The European Physical Journal B-Condensed Matter and Complex Systems, 3(2):271-276.

Cebrat, S., Dudek, M. R., Gierlik, A., Kowalczuk, M., and Mackiewicz, P. (1999). Effect of replication on the third base of codons. Physica A, 265:78-84.

Cebrat, S., Dudek, M. R., and Mackiewicz, P. (1998). Sequence asymmetry as a parameter indicating coding sequence in Saccharomyces cerevisiae genome. Theory in Biosciences, 117:78-89.

Cebrat, S., Dudek, M. R., Mackiewicz, P., Kowalczuk, M., and Fita, M. (1997a). Asymmetry of coding versus non-coding strands in coding sequences of different genomes. Microbial \& Comparative Genomics, 2:259-268.

Cebrat, S., Dudek, M. R., and Rogowska, A. (1997b). Asymmetry in nucleotide composition of sense and antisense strands as a parameter for discriminating open reading frames as protein coding sequences. $J$. Appl. Genet., 38:1-9.

Chib, S. and Greenberg, E. (1995). Understanding the Metropolis-Hastings Algorithm. The American Statistician, 49(4):327-335.

Das, S., Ghosh, S., Pan, A., and Dutta, C. (2005). Compositional variation in bacterial genes and proteins with potential expression level. FEBS Letters, 579:52055210.

Echols, H. and Goodman, M. F. (1991). Fidelity mechanisms in DNA replication. Annu Rev Biochem, 60:477-511.

Frank, A. and Lobry, J. (1999). Asymmetric substitution patterns: a review of possible underlying mutational or selective mechanisms. Gene, 238:65-77.

Freeman, J., Plasterer, T., Smith, T., and Mohr, S. (1998). Patterns of genome organization in bacteria. Science, 279:1827.

Gutierrez, G., Marquez, L., and Martin, A. (1996). Preference for guanosine at first codon position in highly expressed Escherichia coli genes. a relationship with translation efficiency. Nucleic Acids Res., 24:25252528.

Hanawalt, P. C. (1991). Heterogeneity of dna repair at the gene level. Mutation Research/Fundamental and
Molecular Mechanisms of Mutagenesis, 247(2):203211.

Hasegawa, M., Kishino, H., and Yano, T. (1985). Dating of the human-ape splitting by a molecular clock of mitochondrial dna. J Mol Evol, 22(2):160-174.

Hutchinson, F. (1996). Mutagenesis. In Neidhardt, F. C., editor, Escherichia coli and Salmonella. Cellular and molecular biology, pages 749-763. Asm. Press, Washington D.C.

Ikemura, T. (1981). Correlation between the abundance of Escherichia coli transfer RNAs and the occurrence of the respective codons in its protein genes. $J \mathrm{Mol} \mathrm{Biol,}$ 146(1):1-21.

Ikemura, T. (1985). Codon usage and tRNA content in unicellular and multicellular organisms. Mol. Biol. Evol., $2: 1334$.

Kanaya, S., Yamada, Y., Kudo, Y., and Ikemura, T. (1999). Studies of codon usage and trna genes of 18 unicellular organisms and quantification of Bacillus subtilis trnas: gene expression level and species-specific diversity of codon usage based on multivariate analysis. Gene, 238(1):143-155.

Karlin, S., Blaisdell, B. E., and Bucher, P. (1992). Quantile distributions of amino acid usage in protein classes. Protein Eng, 5(8):729-738.

Karlin, S. and Burge, C. (1995). Dinucleotide relative abundance extremes: a genomic signature. Trends Genet, 11(7):283-290.

Karlin, S. and Mrazek, J. (1996). What drives codon choices in human genes? J Mol Biol, 262(4):459-472.

Kowalczuk, M., Gierlik, A., Mackiewicz, P., Cebrat, S., and Dudek, M. (1999). Optimization of gene sequences under constant mutational pressure and selection. Physica A: Statistical Mechanics and its Applications, 273(1):116-131.

Kowalczuk, M., Mackiewicz, P., Mackiewicz, D., Nowicka, A., Dudkiewicz, M., Dudek, M., and Cebrat, S. (2001a). High correlation between the turnover of nucleotides under mutational pressure and the DNA composition. BMC Evol. Biol., 1:13.

Kowalczuk, M., Mackiewicz, P., Mackiewicz, D., Nowicka, A., Dudkiewicz, M., Dudek, M. R., and Cebrat, S. (2001b). DNA asymmetry and the replicational mutational pressure. J. Appl. Genet., 42(4):553-577.

Kreutzer, D. A. and Essigmann, J. M. (1998). Oxidized, deaminated cytosines are a source of $C \rightarrow T$ transitions in vivo. Proc Natl Acad Sci U S A, 95(7):35783582 .

Lagunez-Otero, J. and Trifonov, E. N. (1992). mRNA periodocal infrastructure complementary to the proofreading site in the ribosome. J. Biomol. Struct. Dyn., 10:455-464.

Lindahl, T. (1993). Instability and decay of the primary structure of DNA. Nature, 362(6422):709-715.

Mackiewicz, P., Gierlik, A., Kowalczuk, M., Dudek, M., and Cebrat, S. (1999a). Asymmetry of nucleotide composition of prokaryotic chromosomes. J. Appl. Genet., 40:1-14.

Mackiewicz, P., Gierlik, A., Kowalczuk, M., Szczepanik, D., Dudek, M., and Cebrat, S. (1999b). Mechanisms 
generating long-range correlation in nucleotide composition of the Borrelia burgdorferi genome. Physica A, 273:103-115.

McInerney, J. (1998). Replicational and transcriptional selection on codon usage in Borrelia burgdorferi. Proc. Natl. Acad. Sci. U.S.A., 95:10698-10703.

McLean, M., Wolfe, K., and Devine, K. (1998). Base composition skews, replication orientation, and gene orientation in 12 prokaryote genomes. J. Mol. Evol., 47:691-696.

Mellon, I. and Hanawalt, P. C. (1989). Induction of the Escherichia coli lactose operon selectively increases repair of its transcribed DNA strand. Nature, 342(6245):95-98.

Pan, A., Dutta, C., and Das, J. (1998). Codon usage in highly expressed genes of Haemophillus influenzae and Mycobacterium tuberculosis: translational selection versus mutational bias. Gene, 215:405-413.

Sharp, P. M. and Cowe, E. (1991). Synonymous codon usage in Saccharomyces cerevisiae. Yeast, 7(7):657678.

Shepherd, J. C. (1981). Method to determine the reading frame of a protein from the purine/pyrimidine genome sequence and its possible evolutionary justification. Proc. Natl. Acad. Sci. USA, 78:1596-1600.

Smithies, O., Engels, W. R., Devereux, J. R., Slightom, J. L., and Shen, S. (1981). Base substitutions, length differences and DNA strand asymmetries in the human g gamma and a gamma fetal globin gene region. Cell, 26:345-353.

Tijms, H. (2003). A first course in stochastic processes. John Wiley \& Sons LTD.

Tillier, E. and Collins, R. (2000). The contributions of replication orientation, gene direction, and signal sequences to base composition asymmetries in bacterial genomes. J. Mol. Evol., 50:249-257.

Trifonov, E. N. (1987). Translation framing code and framemonitoring mechanism as suggested by the analysis of mRNA and 16S rRNA nucleotide sequences. J. Mol. Biol., 194:643-652.

Trifonov, E. N. (1992). Recognition of correct reading frame by the ribosome. Biochimie, 74:357-362.

Wakeley, J. (1996). The excess of transitions among nucleotide substitutions: new methods of estimating transition bias underscore its significance. Trends in Ecology and Evolution, 11:158-163.

Wang, J. (1998). The base contents of A, C, G, or U for three codon positions and the total coding sequences show positive correlation. J. Biomol. Struct. Dyn., 16:51-57.

Wong, J. T. and Cedergren, R. (1986). Natural selection versus primitive gene structure as determinant of codon usage. Eur. J. Biochem., 159:175-180.

Zhang, C. T. and Zhang, R. (1991). Analysis of distribution of bases in codon in the coding sequences by a diagrammatic technique. Nucleic Acids Res., 19:63136317. 